\title{
Human Perspective on Affective Robotic Behavior: A Longitudinal Study*
}

\author{
Lilia Moshkina \\ Mobile Robot Laboratory and GVU Center \\ College of Computing, Georgia Institute of Technology \\ Atlanta, GA, USA \\ lilia@cc.gatech.edu
}

\begin{abstract}
Humans are inherently social creatures, and affect plays no small role in their social nature. We use our emotional expressions to communicate our internal state, our moods assist or hinder our interactions on a daily basis, we constantly form lasting attitudes towards others, and our personalities make us uniquely predisposed to perform certain tasks. In this paper, we present a framework under development that combines these four areas of affect to influence robotic behavior, and describe initial results of a longitudinal human-robot interaction study. The study was designed to inform the design of the framework in order to increase ease and pleasantness of human-robot interaction.
\end{abstract}

Keywords: Human-robot interaction, computational models of emotion and personality.

\section{INTRODUCTION}

Humans are inherently social creatures, and apply social rules not only to their interactions with one another, but also to those with non-human animals, and even inanimate objects. This propensity of people to anthropomorphize certain objects has been well established by Nass and his colleagues in an extensive set of experiments [1], which showed that people treat computers as social actors, whether they recognize it or not, and that even minimal cues evoke social responses. Given the predicted pervasiveness of robots in our daily lives, and our social human nature, it is not surprising that many researchers [2, 3] support the paradigm of robots as collaborative partners/companions. Interaction with a partner is, by its nature, social, in which affect plays a vital role. For example, we use our emotional expressions to communicate our internal state, our moods assist or hinder our interactions on a daily basis, we constantly form lasting attitudes towards others, and our personalities make us distinctively ourselves.

We subscribe to the view of robots as partners/companions. To facilitate social interaction between humans and robots, we are developing a framework of affective robotic behavior (TAME, for Traits, Attitudes, Moods and Emotions [4]) that combines the aforementioned emotions, moods, attitudes and personality together, and explicitly defines interactions between them. In order to better inform the design process, we have conducted a longitudinal human-robot interaction study in which 20 participants

\author{
Ronald C. Arkin \\ Mobile Robot Laboratory and GVU Center \\ College of Computing, Georgia Institute of Technology \\ Atlanta, GA, USA \\ arkin@cc.gatech.edu
}

interacted with an autonomous robotic dog (Sony AIBO) in a series of four sessions each, with either an emotional or nonemotional condition. For this study, a partial implementation of Personality and Emotion modules was used. The initial results of the study are reported in the remainder of the paper, along with related work and a brief overview of TAME.

\section{RELATED WORK}

In this section, we present some of the most relevant research in the areas of social robotics and emotion and personality modeling.

Notably, Breazeal's robotic creature Kismet [5] can probably be considered the first socially interactive robot. Although modeled after an infant, it is capable of proto-social responses, providing a user with intuitive means of communication. Kismet also has a motivational system composed of drives and emotions, in which the affect space is defined along three dimensions: arousal, valence and stance.

The approach to robot emotions taken by Velasquez $[6,7]$ is categorical, where the affect space is divided into a number of distinct emotions; Velasquez also extends the role of emotion to range from emotional expression for communication purposes to serving as a determining factor in decision-making processes.

In Arkin et al [8], a somewhat different dimensional emotional model from Breazeal's (the last dimension is confidence instead of stance), serves as part of an extensive motivational system for the Sony robotic dog AIBO, being motivated by ethology and that is directly involved in action selection.

A number of researchers have conducted studies where human attitudes towards robots or animated characters expressing personality or emotion were assessed. In particular, Yan et al [9] encoded Introversion and Extraversion in AIBO, and found that subjects could correctly identify the encoded trait, and preferred to interact with a robot possessing a complementary personality. In a set of studies by a different group [10], it was observed that the personality preference depended on the nature of the task given to the subjects, and that people liked more cheerful robots better, but followed a serious robot's instructions to a greater extent. Finally,

\footnotetext{
${ }^{*}$ This research was funded in part under a seed grant from the GA Tech GVU Center with additional support provided by SAIC.
} 
Tomlinson et al. [11] succeeded in using autonomous expressions of emotion to maintain the believability of animated wolf pups while giving the high-level behavioral control to the user.

Other related research in robotics includes modeling feelings and emotions based on an internal secretion system [12], work on sensing and expression Personalities for humanoid robots [13], mass psychology-based emotional group behavior [14], and utilizing emotional expression in spontaneous, short-term interaction in a museum tour-guide task [15]. Finally, there exists a large body of affect-related work in the domain of animation and autonomous agents, such as Koda's [16] poker-playing agents, the Oz project by Bates, Loyall and Reilly [17], Elliot's "Affective Reasoner" [18], Moffat's [19] Will system, and others.

Fong et al [3] give a comprehensive survey of socially interactive robots, which includes both design methods and an overview of the current research in the area. Picard provides a similar overview of affective computing [20].

\section{ARCHITECTURAL FRAMEWORK}

In the TAME framework, a far larger portion of the affective space is taken into account than most previous work, and the emphasis is not only on modeling each individual component (namely, personality Traits, Attitudes, Moods, and Emotions), but also on explicitly specifying the interactions between these widely time-varying affective phenomena. Although the model takes inspiration from a number of related theories of personality, mood, emotion and attitudes, it is not intended to be a cognitive model of affect and personality, but rather serves as a framework for modeling personality and affect in behavior-based autonomous robotic systems.

\section{A) Overview and Psychological Foundations}

The Personality and Affect module consists of four interrelated components: Personality Traits, Affect-Based Attitudes, Moods, and Emotions. The input into this architectural module consists of relevant perceptual information, such as the categories of visible objects and distances to them (stimuli and their strengths). The output of the module affects currently active behavior by modifying the underlying behavioral parameters, and can influence both low-level, primitive behaviors and higher-level assemblages. Briefly, in our schema-based behavior-based paradigm, a robot's control program consists of a collection of behaviors and coordination mechanisms [21]; primitive behaviors can be combined into behavioral assemblages, and perceptual input produces transitions between them. In the partial integration of this module into MissionLab, a version of AuRA (Autonomous Robot Architecture) [22], each TAME component is implemented at a reactive level as a set of primitive behaviors, but unlike motor schemas, these behaviors do not output motor vectors, but rather change the control parameters for the corresponding active motor schemas. Each component (except for Traits) runs as a separate thread continuously throughout execution The modification of the behavioral control parameters by the personality and emotions modules is described in more detail in $[4,19,23]$. A conceptual view of the overall TAME framework is presented in Figure 1.

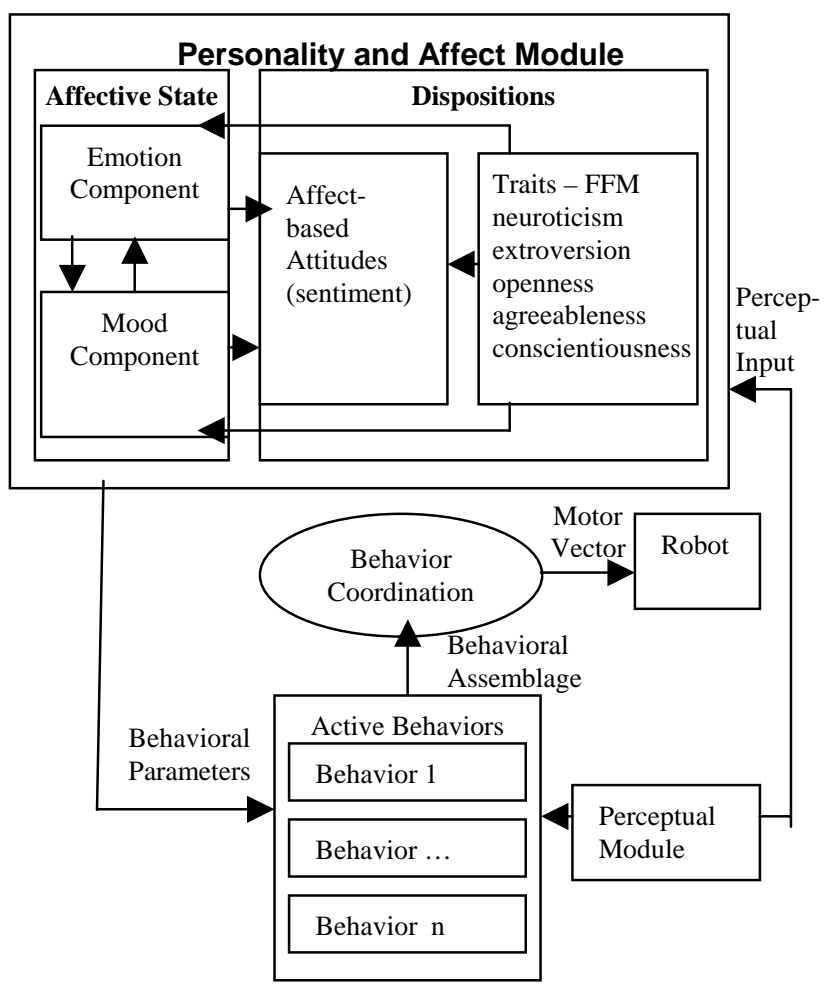

Figure 1: Integrated Model of Personality and Affect (TAME)

Each component occupies a distinct position in the twodimensional space defined by affective duration and specificity [19, 23]. Emotions and moods represent the dynamically changing robot's affective state (high-activation and short-term for emotions, and low-activation and prolonged for moods); where emotions are object-specific, and moods are more generic in their nature. Traits and attitudes are more or less time-invariant, and define general dispositions: in behavior for the case of traits, and to people, objects, or situations for the case of attitudes (Figure 2).

The Five-Factor Model (FFM) of Personality [24] was chosen for the Trait Component due to its generality, stability throughout the lifetime, and applicability to nonhuman animals. The five dimensions of the model are: Openness (O), Agreeableness (A), Conscientiousness (C), Extroversion (E), and Neuroticism (N). Traits in TAME are viewed as constant, operator-defined values prior to execution.

For the Emotion Component, we chose a categorical approach, where emotional phenomena are divided into a set of basic emotions, such as joy, interest, surprise, fear, anger, sadness and disgust. These are recognized by a number of theorists, such as Ekman, Friesen, Izard, Plutchik and Tomkins [25]. Emotions are continuously and dynamically generated as emotion-eliciting stimuli are detected. 
Thayer [26] views moods as a background feeling that persists over time, that emphasizes meaning and enhances or reduces pleasure in our lives. Unlike emotions, this affective state represents a low activation state and is less intense, thus expending less energy and bodily resources [25]. The two categories of moods included in the Mood Component are positive affect and negative affect, which are fairly independent of each other [25].

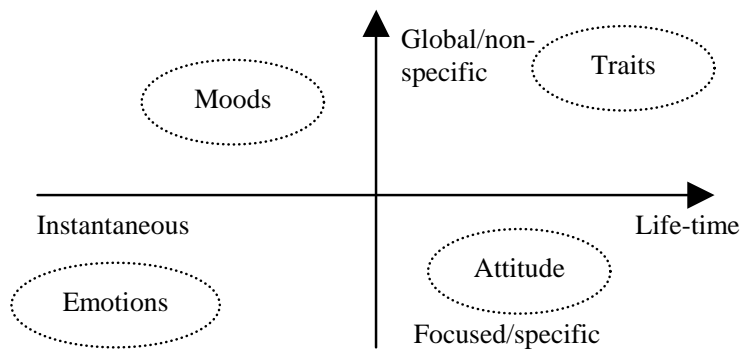

Figure 2: Components Relative to Specificity and Time

Finally, attitudes can be defined as "a general and enduring positive or negative feeling about some person, object or issue" [27], and is relatively time-invariant, object/situation specific, influenced by affect, and result in a certain behavior towards the object.

\section{IMPLEMENTATION}

In order to inform the design of TAME to facilitate interaction between humans and autonomous robots in reallife situations a longitudinal human-robot interaction (HRI) experimental study has been designed and conducted. A partial implementation of the Personality and Emotion modules was provided and adapted to the physical platform: a Sony entertainment robotic dog, AIBO ERS-210A. The choice of the robot was determined by its safety around humans, and the variety of expressive features it possesses, such as variable gaits, movable mouth, ears, tail, and LED display.

\section{A) Basic Features}

The high-level controller was implemented in MissionLab [28], and the lower-level implementation was done in OPEN$\mathrm{R}$ SDK-1.1.3, an open-source programming environment provided by Sony.

When a user uttered a command, it was passed up to a Finite State Acceptor (FSA) in MissionLab by an administrator's key press, and then the processed command was passed on to the low-level controller on the robot.

There were a total of seven commands available: "Stop", "Go Play", "Follow the Ball”, "Kick the Ball”, "Follow Me", "Come to Me", and "Sic' em".

- "Stop" command stops the robot in a suspended state, ready to continue at any moment.

- "Go Play" makes the robot roam around in random directions and perform random stops (no additional user interaction is required for the robot to wander around beyond issuing the command once).
- In "Follow the Ball" mode, the robot looks for a pink ball by walking around in a circle, and moves towards the ball once it is detected; if the ball is lost, looking for the ball is resumed. Color recognition was used to detect the ball.

- "Kick the Ball" is similar to "Follow the Ball" except for when the robot is close enough to the ball to kick it, it performs a kicking motion.

- "Follow Me" command was identical to "Follow the Ball" command, but with a bouquet of artificial flowers used as a prop to follow instead of the ball.

- "Come to Me" is similar to the "Follow Me" command, but the robot stops if it comes within a certain distance to the prop.

- In "Sic' em" command, the robot moves towards the "intruder robot" (ActiveMedia Amigobot) and stops next to it.

The basic set of behaviors was used in the Nonemotional condition, and augmented in the Emotional version via a variety of gaits, movement of ears and tail, LED changes, and minor behavior sequences, described below.

\section{B) Personality}

Although a number of studies have established a link between non-verbal behavior and personality judgments [29, 30], identifying the specific behaviors characteristic to certain personality dimensions has proven to be much harder. Extraversion is the most studied personality trait in this respect, and was reported to positively correlate with an expressive, animated, and expansive behavioral style [29].

To separate personality from emotion, the encoding of personality was used only in the "Go Play" command, and the encoding of emotion was used in all other commands. The personality was determined for the most part by the Extraversion dimension, as well as other traits (for some of the parameters). The parameters modified for the "Go Play" command were as follows: the proportion of time the robot wagged its tail was directly related to its level of Extraversion and Agreeableness; the probability of the robot changing gaits (Slow, Normal, Fast or Crawl) was directly proportional to its level of Extraversion and Openness, and the proportion of time the robot spent walking vs. stopping was inversely proportional to its level of Extraversion, and directly - to its level of Neuroticism. Our intention was to present an energetic, friendly, and curious robot, and therefore the levels of Extraversion, Agreeableness and Openness were set to high, and the level of Neuroticism - to low. The robot also turned its head as it was walking, which contributed to the display of energy and curiosity.

\section{C) Emotion}

A number of sources were used to encode the display of emotions in the robotic dog. We conducted two informal surveys (11 and 21 people each) to find out laymen perceptions of dog emotions; consulted dog behavior literature [31, 32], and used commonsense to adapt the findings to the technical limitations of the platform.

The expressive features used were as follows: three gaits (Normal, Fast - resembled slight jumping, and Crawling - 
somewhat similar to invitation to play/prowling behavior); three tail positions (up, flat, down); tail wagging; two ear positions (up and flat), and red illumination of LED screen.

The emotion expressed was determined by a combination of command type and presence of the command object (stimulus), except for the Sic' em command, where the distance to the intruder robot also played a role. In particular, the following emotions were encoded:

- Alert Interest - during the Kick the Ball command, the dog used the crawling gait; when the pink ball was detected, the ears and tail went up.

- Friendly Interest - during the Follow the Ball command, the dog used the crawling gait; the ears went up and the tail was wagging when the pink ball was detected.

- Alert Joy - during the Follow Me command after the flowers were detected the dog used the Fast walk, the ears and the tail went up.

- Friendly Joy - during the Come To Me command after the flowers were detected the dog used the Fast walk, the ears went up, and the tail was wagging. When the robot was sufficiently close to the flowers, the tail was wagging faster.

- Anger and fear - during the Sic' em command, the robot used the Fast walk, the ears were flat, the tail was up and the LED screen was red until the robot got close to the intruder robot. After that, the red light went out, the tail and the head went down, and the robot backed up using the Crawling gait. A snapshot of the robotic dog "scaring off" the "intruder" by displaying "anger" is shown below (Figure 3).

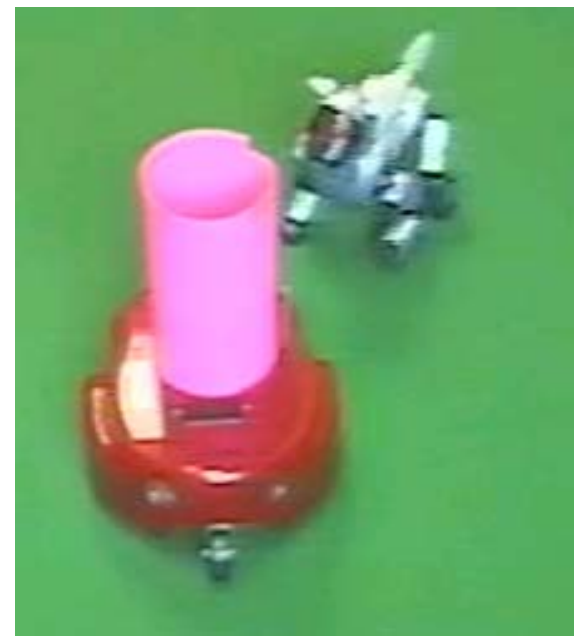

Figure 3: Aibo scares off the Amigobot by displaying “anger”

As different gaits were used in the Emotional and Nonemotional conditions, there were slight differences in performance: it was easier to kick the ball in the Emotional condition, and the Come To Me command performed better in the Non-emotional condition. Additionally, just due to the fact that the robot's appearance resembled that of a puppy, some behaviors not intentionally encoded as emotional (e.g., the head following after object behavior, and the slightly jerky gait) were perceived by some subjects as expressing emotionality.

\section{EXPLORATORY LONGITUDINAL STUDY}

The study was two-fold; the goal was: 1) to find out whether the presence of emotions and personality would increase the perceived ease of use and pleasantness of interaction, and 2) to identify whether the current implementation was sufficient to differentiate between a robot with emotions and personality from one without such. A longitudinal study allowed us to observe the human-robot interaction beyond a single short session, thus letting the "novelty" of interacting with a robot to wear off.

\section{A) Experiment Design and Hypotheses}

The IRB-approved study was set up as a "robot as pet and personal protector" scenario, allowing for the exploration of relevant phenomena in a relatively constrained domain. During each session, the participants were requested to interact with the robot by asking it to perform certain tasks, with a new task introduced at each of the first three sessions. The participants were also encouraged to interact with the dog by petting it, playing with it, addressing it, and otherwise engaging with it if they so chose.

The study followed 1-factor independent design with two conditions: Non-Emotional and Emotional. In the Emotional condition, the robot's basic set of behaviors was augmented with a display of emotions and personality via head, ear and tail position, a variety of gaits, and LED display (as described earlier in the implementation section), whereas in the NonEmotional condition, the basic set of behaviors was left intact.

The following form the hypotheses of this experimental study:

1) Hypothesis 1: The display of emotions and personality will increase the ease of use of autonomous robots;

2) Hypothesis 2: The display of emotions and personality will increase the pleasantness of interaction with autonomous robots and will generate greater attachment to them;

3) Hypothesis 3: The expression of emotions and personality will be more recognizable in the Emotional condition;

4) Hypothesis 4: The display of emotions and personality will result in higher Positive Mood and lower Negative Mood of the participants.

\section{B) Experiment Setup and Procedure}

The study took place in a small quiet office. A Dell Latitude laptop was used to operate the AIBO wirelessly, and a Dell Precision 610 desktop was used to teleoperate the intruder Amigobot via RF. A green carpet was used to specify the borders within which the robot was to be kept by the users; the users had an option of themselves staying on or off the carpet while interacting with the robot, and on or off a wheeled office chair. The video camera was positioned on a desk overlooking the carpet, and the entire interaction between the participants and the robot was captured on video tape within the bounds of the carpet.

In this longitudinal study, the subjects participated in four 20-60 minute interaction sessions. The session duration 
included filling out questionnaires and the interaction with the dog per se, and depended on the willingness of the participants to interact with the robot.

At the beginning of the first session the participants were asked to fill out a demographics questionnaire and a personality questionnaire, then they were introduced to the robot and told that they could make the robot stop or "play" on its own, without requiring user interaction ("Stop" and "Go Play” commands, respectively). Then they were taught two more commands they could give the robotic dog: "follow the ball" and "kick the ball". Each command was first introduced, and then the participants were asked to repeat it three more times. These commands were to be separated by either "Stop" or "Go Play" commands. After the command that was introduced second ("kick the ball”) was repeated three times, the subjects had an option of either continuing to interact with the robot, or completing the experiment by filling out the mood questionnaire (this questionnaire was filled out at the end of each of the four sessions).

In the second session, two new commands ("follow me" and "come to me") were introduced in the same manner as those in the first session. In figure 4, you can see a subject getting the Aibo to perform the "come to me" command (Emotional Condition). After both of the commands were repeated three times, the subjects were asked to interact with the dog for at least five more minutes practicing the commands from this and the previous sessions. After this the participants, again, had an option of continuing the interaction or completing the session.

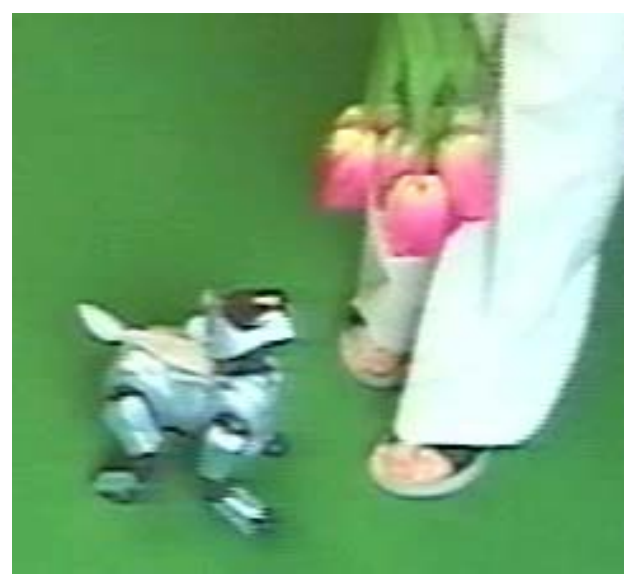

Figure 4: Aibo is performing the "Come to me" command in the Emotional Condition.

During the third session an "intruder robot" was introduced (another small robot, an Active Media robot Amigobot that was used for the purpose of testing the robot's role as a protector. As the Amigobot was teleoperated onto the carpet, the subjects were instructed to hide their props (the pink ball and the flowers) and give the AIBO a "Sic' em" command. After the robotic dog successfully performed the "Sic' em" command, the Amigobot was guided back off the carpet. Subsequent to the initial introduction of this command, the administrator brought out the "intruder robot" three more times, every four minutes. The participants were asked to interact with the dog using any of the commands they know while waiting for the intruder robot to appear. After the last appearance of the Amigobot, the subject could either continue the interaction, or complete the session.

Finally, during the last session the participants were asked to interact with the robot for at least fifteen minutes using any of the commands they know in any order. The "intruder robot" was brought in from time to time (approximately every 3 to 4 minutes) throughout this session. At the end of the fifteen minutes the users could either continue the interaction, or proceed to fill out the rest of the questionnaires. The questionnaires were the mood questionnaire, the personality questionnaire regarding the robotic dog, and the post questionnaire. After all the questionnaires were filled out, some of the participants were given a choice of briefly interacting with the robot in the opposite condition than the one they were exposed to for all prior sessions (e.g., emotional instead of non-emotional).

\section{C) Measures}

Evaluation was performed using both introspection (questionnaires) and observation (videotapes analysis) methods with respect to the aforementioned study hypotheses. The videotape analysis is pending, and will be presented in a subsequent report.

The post questionnaire was designed to assess hypotheses 1-3. It consisted of six 5-point Likert scale questions with three subquestions, with "Strongly Agree" anchored at 5, and "Strongly Disagree" anchored at 1 . The questions were as follows:

1) It was easy to get the robotic dog perform the commands;

2) It was easy to understand whether the robotic dog was performing the command or not;

3) The robotic dog showed emotional expressions;

4) The robotic dog had a personality of its own;

5) With every session, I was getting more attached to the dog;

6) Overall, I enjoyed the interaction with the robotic dog.

If the participants answered "Agree" or "Strongly Agree" to question 3 or 4 , they were also asked to answer questions 3a,b and 4a, respectively. The subquestions were as follows:

3a) Emotional expressions exhibited by the dog made the interaction more enjoyable;

3b) Emotional expressions exhibited by the dog made the interaction easier;

4a) I enjoyed interacting with the robot, partly because it possessed some personality.

Questions 1, 2 and $3 b$ were used as measures for Hypothesis 1; questions 3a, 4a, 5, and 6 were used as measures for Hypothesis 2, and questions 3 and 4 served as measure for Hypothesis 3.

To analyze Hypothesis 4, the answers from the PANAS$\mathrm{T}$ (positive/negative emotionality measure, or "mood") questionnaire [33] were averaged across the four sessions. 
Finally, the participants were also asked to fill out two personality questionnaires: the one regarding their personality was filled out at the beginning of the first session, and the one regarding the robotic dog's personality - at the end of the last session. Both were based on a brief version of Goldberg's Unipolar Big-Five Markers (personality questionnaire) [34]; the dimensions assessed were Extraversion, Agreeableness, Conscientiousness, Emotional Stability (Neuroticism) and Openness to Experience/Intellect. Pearson's Correlation analysis was conducted on the participants' and the robot's personality to identify whether the subjects projected their own personality on the robot, but none of the dimensions had significant correlations.

\section{D) Participants}

A total of 20 people participated in the study: 10 males and 10 females, distributed equally between the two conditions. The subjects varied widely in the demographics according to age (from between 20 and 30 to over 50 years old), their educational level and backgrounds (from High School diploma to working on a Ph.D., with majority having either a Bachelor's or Master's degrees), and computer experience. Most of the participants had owned pets at some point in their lives (18 out of 20), and had either no or very limited robot interaction experience (only 2 out of 20 had interacted with mobile or entertainment robots prior to the study).

\section{E) Analysis and Results}

1-tailed Independent Samples T-tests were conducted on all the measures. The graphs (except for Figure 6) display the plots of Means and Means+-Standard Error.

\section{1) Hypothesis 1}

The result of the analysis of question 1 was statistically significant ( $\mathrm{M}_{\text {non-emotional }}=3.7, \mathrm{M}_{\text {emotional }}=4.5, \mathrm{~F}=0.02$, $\mathrm{p}<0.004)$. The result is presented visually in Figure 5:

Although the analysis shows that the participants found it easier to get the robotic dog to perform the commands in the Emotional condition, this result is confounded by the slight performance differences for two of the commands, and therefore this result is potentially due to two factors: the performance difference and the emotionality condition; further study would be needed to disambiguate it.

There was no significant effect of Emotionality on the answers to question 2. As for question $3 \mathrm{~b}$, the average answer was 3.54 (between neutral and agree), suggesting that those who thought that the robot displayed emotional expressions (5 out of 10 in the Non-Emotional condition, and 8 out of 10 in the Emotional condition), considered emotional expression helpful rather than not in making the interaction easier, although only slightly.

\section{2) Hypothesis 2}

The analysis of the answers to questions 5 and 6 didn't show any significant differences between the two conditions with respect to the overall pleasantness of interaction and the degree of attachment to the robot. However, those who thought that the robot displayed emotions and/or personality ( 6 out of 10 in each condition), found that both emotion and personality made their interaction with the robotic dog more enjoyable: the average answer for question 3a was 4.46, and for question 4a was 4.25. These results are displayed graphically in Figure 6.

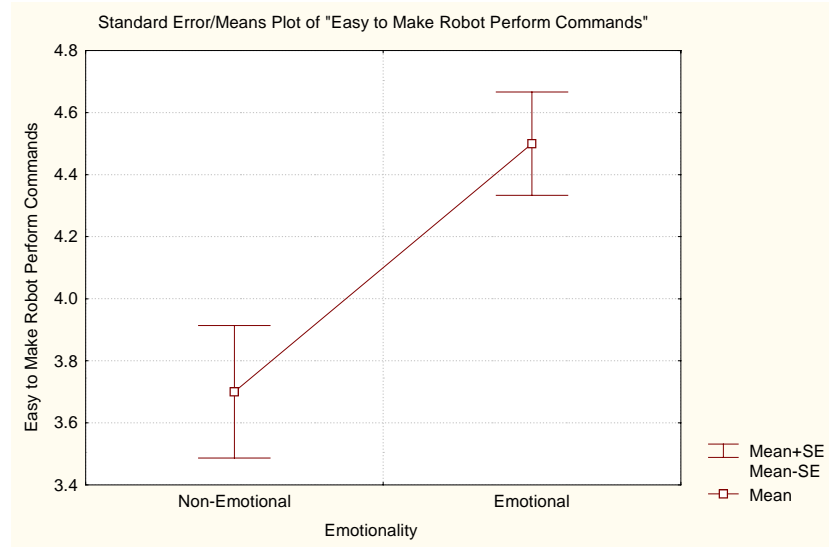

Figure 5: Standard Error/Means Plot for "Ease of Performing Command"; it was easier to make the robotic dog perform the commands in the emotional condition.

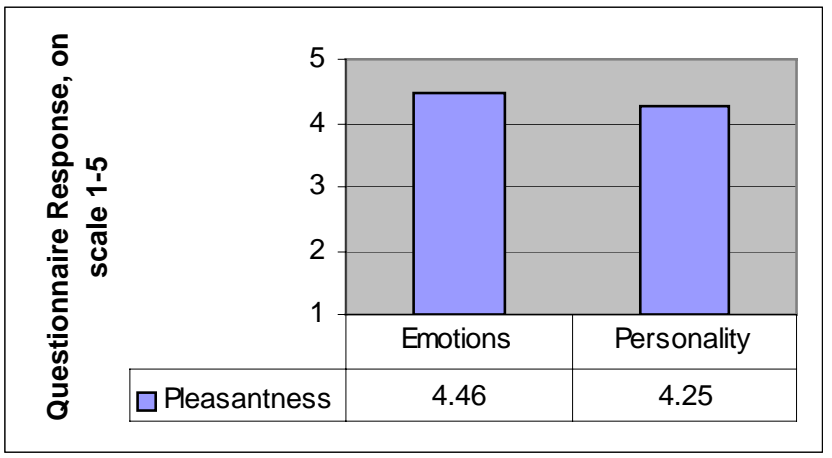

Figure 6: Average of the answers to questions 3a and 4a: the subjects thought the perceived robotic emotions or personality made their interaction more

\section{3) Hypothesis 3} enjoyable.

There was no significant difference between the two conditions regarding perceived emotional and personality display (questions 3 and 4). However, 2-factor ANOVA on Gender and Emotionality resulted in a significant main effect of Gender on the answer to question 3: display of emotions $\left(\mathrm{M}_{\text {female }}=3.8, \quad \mathrm{M}_{\text {male }}=2.5, \mathrm{~F}=4.829, \quad \mathrm{p}<0.043\right)$. The following graph presents this result (Figure 7).

As was mentioned before, the base (non-emotional behavior) could have been perceived as emotional by some participants due to the fact that the platform itself was designed to elicit emotional response from humans. This could partly account for the fact that 5 out of 10 people in the Non-emotional condition perceived the robot as displaying emotionality.

In a related study, Yan et al [9] could manipulate the encoded Introversion and Extraversion dimensions of personality successfully, suggesting that people do pick up nonverbal and verbal personality cues in robots, even though this was not confirmed in our study. One of the reasons we 
did not see any difference in perceived personality between the two conditions in our study may lie in the fact that the personality was encoded only in one task, "Go Play", and the subjects did not have sufficient exposure to its display. Another reason could be that our encoding was broader and less specific than in the aforementioned study, and thus did not produce the expected effect.

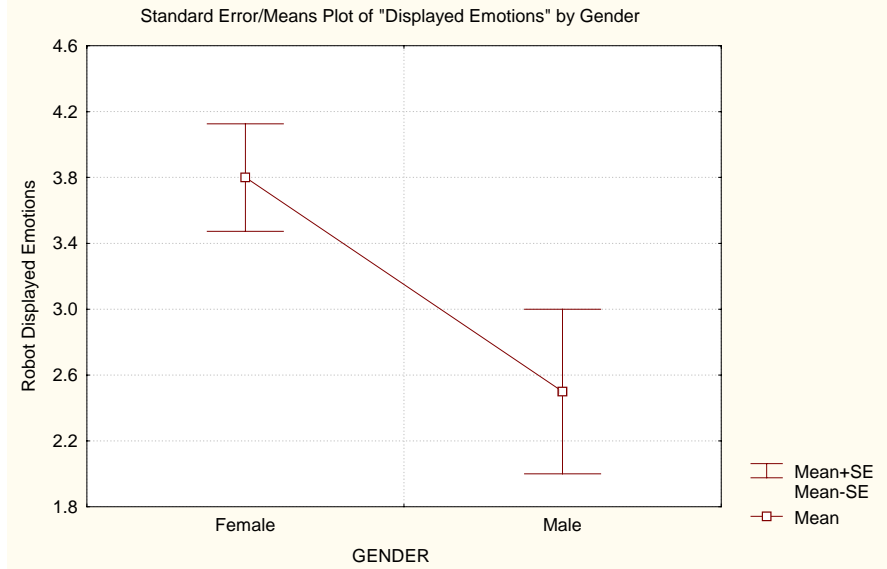

Figure 7: Standard Error/Means Plot for "Displayed Emotions” by Gender: Women perceived the robot more emotional than men, regardless of the condition.

\section{4) Hypothesis 4}

Although there was no significant result of Emotionality on the Positive Mood, the Negative Mood was significantly lower in the Emotional condition $\left(\mathrm{M}_{\text {non-emotional }}=13.9\right.$, $\left.\mathrm{M}_{\text {emotional }}=12.125, \mathrm{~F}=6.462, \mathrm{p}<0.048\right)$. See figure 8 for the plot.

\section{5) Other Observations}

We have also observed that the subjects in the Emotional condition rated the robotic dog higher on the dimensions of Conscientiousness and Openness, and in the 1-tailed independent samples T-test this difference was statistically significant $(\mathrm{p}<.034$, and $\mathrm{p}<.026$, respectively). The last finding, however, may also be convoluted by the aforementioned difference in the performance.

\section{F) Discussion}

A number of interesting and encouraging observations were made. First, those participants who believed that the robot displayed emotions and/or personality also believed that these features made their interaction more pleasant. This is encouraging, as it suggests that people value expression of emotion and personality in their interaction with an autonomous entertainment robot. Second, the reduced level of negative mood in the subjects in the Emotional condition may also suggest that affective behavior contributes to the quality of interaction. Finally, women were found to be more attuned to emotional expressions and more ready to attribute emotions to the robot than men, which should be taken into consideration for systems adapted to groups with genderbiased compositions.

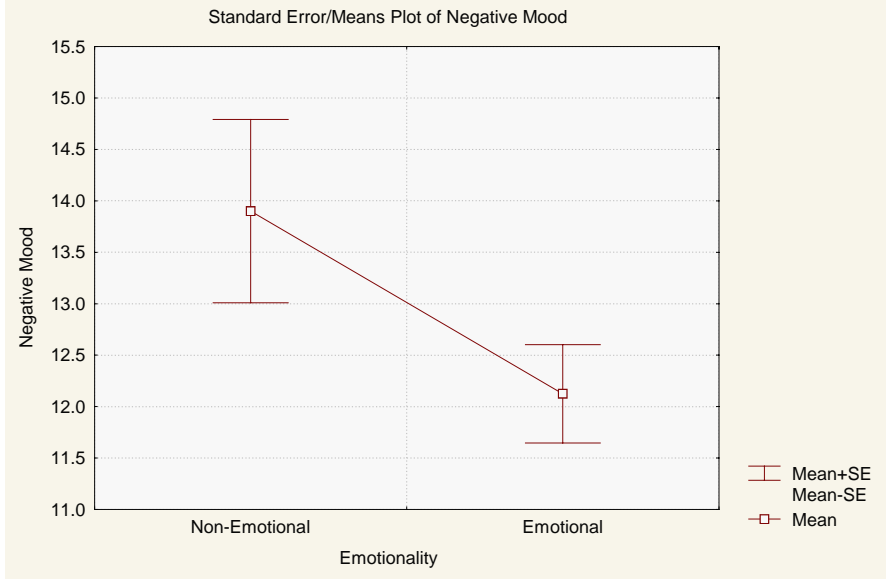

Figure 8: Standard Error/Means Plot for Negative Mood: on average, the subjects experience less negative mood in the Emotional Condition

There are a number of reasons why no difference was observed in the post questionnaire in the area of pleasantness of interaction and perception of emotions and personality. First, the sample size, due to the technical difficulty of the study, was rather small. Second, the chosen physical platform, designed for entertainment, may be a more decisive factor than the differences in the robot behavior. Finally, some researchers [35] are of an opinion that self-report data tends to be unreliable. In this case, the upcoming video analysis may reveal additional insights. From anecdotal evidence, the majority of those who interacted with the robot in the Emotional condition, and had a chance to interact with the non-emotional robot at the end of the study, showed a clear preference for the emotional robot, and vice versa.

\section{CONCLUSIONS AND FUTURE WORK}

In this paper, we described a longitudinal humaninteraction study in which 20 human participants interacted with a Sony robotic dog Aibo in two conditions: Emotional and Non-emotional; and presented the initial results of the study. Some important insights were gleaned: those who believed that the robot displayed emotions and/or personality also believed that these features made their interaction more pleasant; the Negative mood was reduced in the Emotional condition; and women were more susceptible to emotional cues and more willing to attribute emotions to the robot. Additionally, we confirmed that the platform used may significantly affect the human perception, and we are planning to use a more neutral platform for future experiments. Further analysis on this data set will be performed, and the results will be presented in a subsequent report.

\section{ACKNOWLEDGEMENT}

The authors would like to thank Katherine Lau for her tremendous help in preparing and administering the study.

\section{REFERENCES}

1. Nass, C., Moon, Y., Morkes, J., Kim, E-Y., Fogg, B.J., Computers are social actors: A review of current research, in Moral and ethical issues in human-computer interaction, B. Friedman, Editor. 1997, CSLI Press: Stanford, CA. 
2. Breazeal, C., Brooks, A., Gray, J., Hoffman, G., Kidd, C., Lee, H., Lieberman, J., Lockerd, A., Chilongo, D., Tutelage and Collaboration for Humanoid Robots. International Journal of Humanoid Robotics, 2004. 1(4): p. 315-348.

3. Fong, T., Nourbakhsh, I., Dautenhahn, K., A Survey of Socially Interactive Robots. Robotics and Autonomous Systems, 2003. 42: p. 143-166.

4. Moshkina, L., Arkin, R.C., On TAMEing Robots. Interanational Conference on Systems, Man and Cybernetics, 2003.

5. Breazeal, C., Scassellati, B., How to Build Robots that Make Friends and Influence People. IROS, 1999: p. 858-863.

6. Velásquez, J., When Robots Weep: Emotional Memories and DecisionMaking. 15th National Conference on Artificial Intelligence, 1998.

7. Velasquez, J.D., An Emotion-Based Approach to Robotics. IROS, 1999: p. 235-240.

8. Arkin, R.C., Fujita, M., Takagi, T., Hasegawa, R., An Ethological and Emotional Basis for Human-Robot Interaction. Robotics and Autonomous Systems, 2003. 42(3-4).

9. Yan, C., Peng, W., Lee, K.M., Jin, S., Can robots have personality?An empirical study of personality manifestation, social responses, and social presence in human-robot interaction. 54th Annual Conference of the International Communication Association, 2004.

10. Goetz, J., Kiesler, S., Cooperation with a robotic assistant. ACM CHI'2002 Conference on Human Factors in Computing Systems, 2002: p. 578-579.

11. Tomlinson, B., Downie, M., Berlin, M., Gray, J., Lyons, D., Cochran, J., Blumberg, B., Leashing the AlphaWolves: mixing user direction with autonomous emotion in a pack of semi-autonomous virtual characters. ACM SIGGRAPH/Eurographics symposium on Computer animation, 2002: p. 7-14.

12. Ogata, T., Sugano, S., Emotional Communication between Humans and the Autonomous Robot which has the Emotion Model. ICRA, 1999: p. 3177-3182.

13. Miwa, H., Takanishi, A., Takanobu, H., Experimental Study on Robot Personality for Humanoid Head Robot. IROS, 2001: p. 1183-1188.

14. Ishihara, H., Fukuda, T., Performance of Emotional Group Robotic System using Mass Psychology. IROS, 1997: p. 1445 -1450.

15. Schulte, J., Rosenberg, C., Thrun, S., Spontaneous, Short-term Interaction with Mobile Robots. ICRA, 1999: p. 658-663.

16. Koda, T., Agents with faces: a study on the effects of personification of software agents, in MS Thesis. 1996, MIT: Cambridge, MA.

17. Bates, J., Loyall, A.B., Reilly, W.S., An Architecture for Action, Emotion, and Social Behavior, in School of Computer Science CMU-CS92-144. 1992, CMU: Pittsburgh, PA.
18. Elliott, C., Siegle, G., Variables Influencing the Intensity of Simulated Affective States. AAAI Spring Symposium on Reasoning about Mental States: Formal Theories and Applications, 1993: p. 58-67.

19. Moffat, D., Personality Parameters and Programs. Creating Personalities for Synthetic Actors, 1997: p. 120-165.

20. Picard, R.W., Affective Computing. 1997, The MIT Press: Cambridge, MA.

21. Arkin, R., Motor-Schema based Mobile Robot Navigation. International Journal of Robotics Research, 1989. 8(4): p. 92-112.

22. Arkin, R., Balch, T., AuRA: Principles and Practice in Review. Journal of Experimental and Theoretical Artificial Intelligence, 1997. 9(2): p. 175-189.

23. Matthews, G., Derryberry, D., Personality and Emotion: Cognitive Science Perspectives. Advances in Personality Psychology, 2000. 1: p. 199-237.

24. McCrae, R.R., Costa, P.T., Toward a new generation of personality theories: theoretical contexts for the five-factor model. Five-Factor Model of Personality, 1996: p. 51-87.

25. Watson, D., Clark, L.A., Tellegen, A., Mood and Temperament. 2000: The Guilford Press.

26. Thayer, R.E., The Origin of Everyday Moods. 1996: Oxford University Press.

27. Breckler, S.J., Wiggins, E.C., On Defining Attitude and Attitude Theory: Once More with Feeling, in Attitude Structure and Function, A.R. Pratkanis, Breckler, S.J., Greenwald, A.G., Editor. 1989, Lawrence Erlbaum Associates. p. 407-429.

28. MacKenzie, D.C., Arkin, R.C., Cameron, J.M., Multiagent Mission Specification and Execution. Autonomous Robots, 1997. 4(1): p. 29-52.

29. Lippa, R., The Nonverbal Display and Judgement of Extraversion, Masculinity, Femininity, and Gender Diagnosticity: A Lens Model Analysis. Journal of Research in Personality, 1998. 32: p. 80-107.

30. Riggio, E.R., Lippa, R., Salinas, C., The Display of Personality in Expressive Movement. Journal of Personality Research, 1990. 24: p. 1631.

31. Buytendijk, F., The Mind of the Dog. 1973, Arno Press: New York.

32. Fox, M., The Dog: Its Domestication and Behavior. 1978, Garland: New York.

33. Watson, D., Clark, L.A., Tellegen, A., Development and validation of brief measures of positive and negative affect: The PANAS scales. Journal of Personality \& Social Psychology, 1998. 56(6): p. 1063-1070.

34. Saucier, G., Mini-Markers: a Brief Version of Goldberg s Unipolar Big-Five Markers. Journal of Personality Assessment, 1994. 63(3): p. 506-516.

35. Zajonc, R.B., Emotions, in The Handbook of Social Psychology, D.T. Gillbert, Fiske, S.T., Lindzey, G., Editor. 1998. 